\title{
Recommendation of low-dose CT in the detection and management of COVID-2019
}

\author{
Zhen Kang ${ }^{1} \cdot \mathrm{Xu} \mathrm{Li}^{1} \cdot$ Shuchang Zhou ${ }^{1}$ \\ Received: 25 February 2020 / Revised: 10 March 2020 / Accepted: 13 March 2020 / Published online: 19 March 2020 \\ (C) European Society of Radiology 2020
}

CT imaging plays an important role in the diagnosis of COVID-2019, especially in the early stages of the disease or with a low viral load; swab tests may be negative [1]. Fang $\mathrm{Y}$ et al reported a sensitivity of $98 \%$ for chest CT diagnosis of COVID-2019, while RT-PCR serves as the gold standard; its early sensitivity was only $71 \%$ [2]. The vast majority, $98 \%$, show bilateral lung involvement. The typical chest CT findings of non-ICU patients are bilateral ground-glass opacity and sub-segmental consolidation, while the typical chest CT findings of ICU patients are bilateral multi-lobe and sub-segmental consolidation [3]. The affected lungs are mostly located in the peripheral zone. Other imaging features include linear opacities, "crazy-paving" pattern, and the "reverse halo" sign [4]. According to the COVID-2019 pneumonia diagnosis and treatment scheme of the People's Republic of China (trial version 5), imaging features can upgrade suspected cases of new coronavirus pneumonia to clinical diagnosis.

In the face of the sudden, rapid progress of the outbreak, patients need close clinical observation. This includes recurrent $\mathrm{CT}$ to determine the progression or absorption of lesions in the lung within a short period of time, especially for severe cases. Inflammatory absorption of the lungs on CT imaging is also one of the criteria for release from isolation and discharge in the guidelines from the first edition to the sixth edition. Actually, the number of CT scans ranges from 3 to 6 [5] within a short period of time. Even healthy people may get one CT or even two to ensure they do not have COVID-2019. Medical radiation exposure is therefore a concern, because the population is made of patients of all ages, including young individuals, infants, and puerperae, for whom the radiation dose is even a greater concern.

With the advantages of an improved detector, highpitch settings, lower tube voltage $(80-100 \mathrm{kVp})$ and current (10-25 mAs), iterative reconstruction, and dosereduction options, it is now feasible to minimize the radiation dose. We implemented a low-dose scanning protocol that reduced the patient's dose to $1 / 8$ to $1 / 9$ of the standard dose. In addition to all the abovementioned reduction dose strategy, we used energy spectrum tinfiltering technology (Sn100 low-dose protocol, Turbo Flash, SOMATOM Force, Siemens Healthineers).

The dose-length product (DLP) and effective dose (ED) were $14.5 \mathrm{mGy} \mathrm{cm}$ and $0.203 \mathrm{mSv}$ compared with $129.1 \mathrm{mGy} \mathrm{cm}$ and $1.8074 \mathrm{mSv}$ in a standard-dose protocol, without significant sacrifice of signal-to-noise (SNR) or contrast-to-noise (CNR) ratios (Table 1 and Fig. 1). Thus,

Shuchang Zhou

zhoushuchang2020@163.com

1 Department of Radiology, Tongji Hospital, Tongji Medical College, Huazhong University of Technology and Science, Jiefang Avenue, \#1095, Wuhan 430030, China 
Table 1 Parameters of protocols of low-dose vs. standard-dose

\begin{tabular}{llc}
\hline Parameters & Low-dose protocol & Standard-dose protocol \\
\hline Kv & Sn100 & 100 \\
mAs/ref. & $112 / 96$ & $85 / 62$ \\
CTDIvol*, mGy & $0.39 \mathrm{~L}$ & $3.44 \mathrm{~L}$ \\
DLP, mGy cm & 14.5 & 129.1 \\
TI, s & 0.25 & 0.5 \\
cSL, mm & 0.6 & 0.6 \\
ED, mSv & 0.203 & 1.8074 \\
SNR & 0.47 & 0.78 \\
CNR & 1.09 & 3.79 \\
\hline
\end{tabular}

$c S L$, collimation slice; $T I$, inversion time

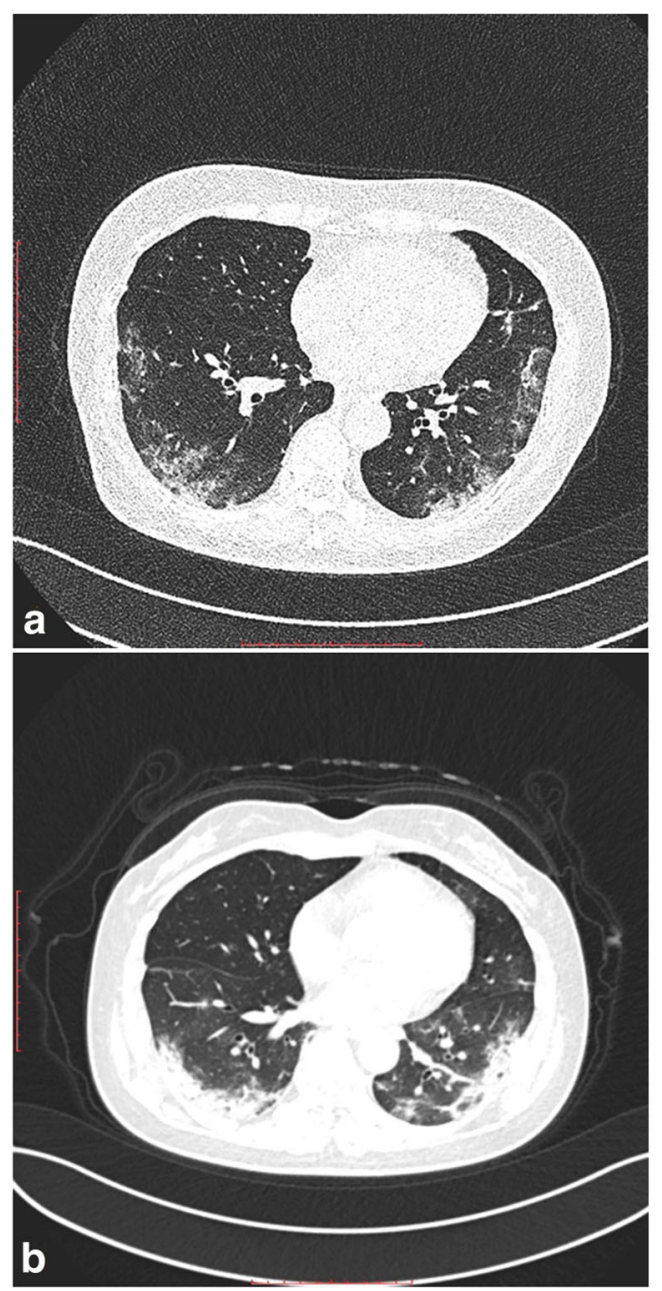

Fig. 1 COVID-2019 pneumonia was diagnosed in two adults of similar height, body size, and age, with typical CT manifestation of ground-glass opacification in the peripheral zone. Excellent image quality is obtained both in low-dose protocol (a) and standard-dose protocol (b) the cumulative radiation dose of serial CT examinations during the acute period might be inferior or equivalent to that of a conventional CT.

As the main expected lesions are within the lung parenchyma, a low-dose protocol is certainly the way to go.

Funding information The authors state that this work has not received any funding.

\section{Compliance with ethical standards}

Guarantor The scientific guarantor of this publication is Shuchang Zhou.

Conflict of interest The authors of this manuscript declare no relationships with any companies, whose products or services may be related to the subject matter of the article.

Statistics and biometry No complex statistical methods were necessary for this paper.

Informed consent Written informed consent was waived by the Institutional Review Board.

Ethical approval Institutional Review Board approval was obtained.

Methodology

- Not applicable

\section{References}

1. Xie X, Zhong Z, Zhao W, Zheng C, Wang F, Liu J (2020) Chest CT for typical 2019-nCoV pneumonia: relationship to negative RT-PCR testing. Radiology. https://doi.org/10.1148/radiol.2020200343: 200343

2. Fang Y, Zhang H, Xie J et al (2020) Sensitivity of chest CT for COVID-19: comparison to RT-PCR. Radiology. https://doi.org/10. 1148/radiol.2020200432:200432

3. Huang C, Wang Y, Li X et al (2020) Clinical features of patients infected with 2019 novel coronavirus in Wuhan, China. Lancet 395:497-506

4. Bernheim A, Mei X, Huang M et al (2020) Chest CT findings in coronavirus disease-19 (COVID-19): relationship to duration of infection. Radiology. https://doi.org/10.1148/radiol.2020200463: 200463

5. Pan F, Ye T, Sun P et al (2020) Time course of lung changes on chest CT during recovery from 2019 novel coronavirus (COVID-19) pneumonia. Radiology. https://doi.org/10.1148/radiol.2020200370: 200370

Publisher's note Springer Nature remains neutral with regard to jurisdictional claims in published maps and institutional affiliations. 